\title{
A Novel Iterative Method for Solving Systems of Fractional Differential Equations
}

\author{
E. Hesameddini and A. Rahimi \\ Department of Mathematics, Faculty of Basic Sciences, Shiraz University of Technology, Shiraz, Modarres Bolvd. P.O. Box, \\ 71555-313, Iran \\ Correspondence should be addressed to E. Hesameddini; hesameddini@sutech.ac.ir
}

Received 26 January 2013; Accepted 9 July 2013

Academic Editor: D. R. Sahu

Copyright (C) 2013 E. Hesameddini and A. Rahimi. This is an open access article distributed under the Creative Commons Attribution License, which permits unrestricted use, distribution, and reproduction in any medium, provided the original work is properly cited.

\begin{abstract}
The Reconstruction of Variational Iteration Method (RVIM) technique has been successfully applied to obtain solutions for systems of nonlinear fractional differential equations: $D_{*}^{\alpha} x_{i}(t)=N_{i}\left(t, x_{1}, \ldots, x_{n}\right), x_{i}^{(k)}=c_{k}^{i}, 0 \leq k \leq\left[\alpha_{i}\right], 1 \leq i \leq n$, where $D_{*}^{\alpha}$ denote Caputo fractional derivative. The RVIM, for differential equations of integer order is extended to derive approximate analytical solutions for systems of fractional differential equations. Advantage of the RVIM, is simplicity of the computations and convergent successive approximations without any restrictive assumptions or transform functions. Some illustrative examples are given to show the validity of this method for solving linear and nonlinear systems of fractional differential equations.
\end{abstract}

\section{Introduction}

In recent years, the fractional differential equations have received remarkable attention. Differential equations of fractional order have been found to be effective to describe some physical phenomena such as rheology, fluid flow, diffusive transport, electrical network, and electromagnetic theory [1-4]. There are different methods to solve the fractional differential equations. Some of the recent analytic methods for solving a system of nonlinear fractional differential equations are the Adomian decomposition method (ADM) [5-8], differential transform method [9], and Variational Iteration method (VIM) [10].

The differential transform method was first applied in engineering in 1986 [11]. Ertürk and Momani introduced a new application of the differential transform method to provide an approximate solution for systems of fractional differential equations [9]. For this propose He developed the Variational Iteration Method (VIM) in 1999 [10]. In this method, the solution is approximated at first iteration by using the initial conditions. A correction functional is established by the general Lagrange multiplier which can be identified optimally via the variational theory. Although a number of useful attempts have been made to solve fractional equations via the VIM, the problem has not yet been completely resolved; that is, most of the previous work avoid the term of fractional derivative and handle them as restricted variations and they cannot identify the fractional Lagrange multipliers explicitly in the correction function. Hesameddini and Latifizadeh proposed a new alternative approach to derivation of the Variational Iteration formulations using the Laplace transform for solving linear and nonlinear ordinary differential equations which was called the Reconstruction of Variational Iteration Method [12]. This method does not use a Lagrange multiplier.

Partial differential equations of fractional order are often very complicated to be exactly solved and even if an exact solution is obtainable, the required calculations may be too complicated to be practical, or it might be difficult to interpret the outcome.

In this work, we extend the Reconstruction of Variational Iteration Method to solve systems of fractional differential equations. The aim of this work is to present an alternative approach based on RVIM to find the solution for linear and nonlinear system of fractional differential equations. 
The efficiency and accuracy of RVIM are demonstrated through several test examples.

\section{Preliminaries and Notations}

In this section, some necessary definitions and mathematical preliminaries of the fractional calculus theory which are used further in this paper will be presented.

Definition 1. Let $C[a, b]$ denotes the space of continuous functions defined on $[a, b]$ and $C^{n}[a, b]$ denotes a class of all real valued functions defined on $[a, b]$ which have continuous $n$th order derivative.

Definition 2. Let $f \in C[a, b]$ and $\alpha \geq 0$; then the expression

$$
I^{\alpha} f(t)=\frac{1}{\Gamma(\alpha)} \int_{0}^{t}(t-\tau)^{\alpha-1} f(\tau) d \tau,
$$

is called the Riemann-Liouville integral of order $\alpha$.

Definition 3. The fractional derivative of $f(x)$ in the Caputo sense is defined as

$$
\begin{aligned}
& D_{*}^{\alpha} f(t) \\
& = \begin{cases}I^{m-1} f^{(m)}(t) & \frac{1}{\Gamma(m-\alpha)} \\
\times \int_{0}^{t}(t-\tau)^{m-\alpha-1} f^{(m)}(\tau) d \tau, & m-1<\alpha<m, \\
\frac{d^{m}}{d x^{m}} f(t), & \alpha=m,\end{cases}
\end{aligned}
$$

for $m \in N$ and $f \in C^{n}[a, b]$.

Note that

$$
\begin{gathered}
I^{\alpha} I^{\beta} f(t)=I^{\alpha+\beta} f(t), \quad \alpha, \beta \geq 0, \\
I^{\alpha} t^{\gamma}=\frac{\Gamma(\gamma+1)}{\Gamma(\gamma+\alpha+1)} t^{\gamma+\alpha}, \quad \alpha>0, \gamma>-1, t>0 .
\end{gathered}
$$

Definition 4. Given a function $f(t)$ defined for all $t \geq 0$, the Laplace transform of $f$ is the function $F$ defined as follows:

$$
F(s)=l\{f(t ; s)\}=\int_{0}^{\infty} e^{-s t} f(t) d t,
$$

for all values of $s$ for which the improper integral converges.

Definition 5. The function $f$ is said to be of exponential order as $t \rightarrow+\infty$ if there exist nonnegative constants $M, c$, and $T$ such that

$$
|f(t)| \leq M e^{c T} \text { for } t \geq T .
$$

Definition 6. The function $f(t)$ is said to be piecewise continuous on the bounded interval $a \leq t \leq b$ provided that $[a, b]$ can be subdivided into finitely many abutting subintervals in such a way that
(1) $f$ is continuous in the interior of each of these subintervals,

(2) $f(t)$ has a finite limit as $t$ approaches each endpoint of each subinterval from the interior.

Theorem 7 (existence of the laplace transforms). If the function $f$ is piecewise continuous for $t \geq 0$ and is of exponential order as $t \rightarrow+\infty$, then its Laplace transform $F(s)$ exists. More precisely, if $f$ is piecewise continuous and satisfies the condition (5), then $F(s)$ exists for all $s>c$.

Definition 8. Let the functions $f(t)$ and $g(t)$ be defined for $t \geq 0$; then the convolution of them is denoted by $(f * g)(t)$ and is defined as the following integral:

$$
(f * g)(t)=\int_{0}^{t} f(\tau) g(t-\tau) d \tau .
$$

In other words, if $l\{f(t)\}=F(s), l\{g(t)\}=G(s)$, then $l\{(f *$ $g)(t)\}=F(s) G(s)$. Or equivalently, $l\left\{\int_{0}^{t} f(\tau) g(t-\tau) d \tau\right\}=$ $F(s) G(s)$. Therefore, the inverse Laplace transform will be defined as

$$
l^{-1}\{F(s) G(s)\}=\int_{0}^{t} f(\tau) g(t-\tau) d \tau .
$$

Definition 9. The Laplace transform of the Caputo fractional derivative $D_{*}^{\alpha} f(t)$ is given by

$$
l\left\{D_{*}^{\alpha} f(t) ; s\right\}=s^{\alpha} F(s)-\sum_{k=0}^{m-1} s^{(\alpha-k-1)} f^{(k)}\left(0^{+}\right),
$$

where $F(s)=l\{f(t) ; s\}, m-1 \leq \alpha<m$.

\section{System of Fractional Differential Equations and Reconstruction of Variational Iteration Method (RVIM)}

Consider a system of fractional differential equations as follows:

$$
\begin{gathered}
D_{*}^{\alpha} x_{1}(t)=N_{1}\left(t, x_{1}, x_{2}, \ldots, x_{n}\right), \\
D_{*}^{\alpha} x_{2}(t)=N_{2}\left(t, x_{1}, x_{2}, \ldots, x_{n}\right), \\
\vdots \\
D_{*}^{\alpha} x_{n}(t)=N_{n}\left(t, x_{1}, x_{2}, \ldots, x_{n}\right),
\end{gathered}
$$

where $N_{i}$ 's are linear/nonlinear functions of $t, x_{1}, x_{2}$, $\ldots, x_{n}, D_{*}^{\alpha_{i}}$ is the derivative of $x_{i}$ with order of $\alpha_{i}$ in the sense of Caputo and $m-1 \leq \alpha_{i}<m$ with $m \geq 1$, subjected to the initial conditions:

$$
x_{i}^{(k)}=c_{k}^{i} \quad 0 \leq k \leq\left[\alpha_{i}\right], 1 \leq i \leq n .
$$

Equation (10) can be rewritten down as a correction function in the following way:

$$
D_{*}^{\alpha} x_{i}(t)=N_{i}\left(t, x_{1}, \ldots, x_{n}\right), \quad i=1, \ldots, n .
$$


Therefore, the approximate solution can be reached as follows:

$$
x_{i}(t)=\lim _{n \rightarrow \infty} x_{i}^{n}(t), \quad i=1, \ldots n,
$$

where $x_{i}^{n}$ indicates $n$th approximation of $x_{i}$ and $x_{i}^{0}$ is $x_{i}(0)+$ $t x_{i}^{\prime}(0)+\cdots+t^{n} x_{i}^{(n)}(0) / n$ ! where $x_{i}^{(k)}(0), k=0,1, \ldots, n$ are substituted by initial condition of the main problem.

Taking Laplace transform to both sides of (11) in the usual way and using the homogenous initial conditions (i.e., artificial initial conditions equal to zero), the result can be obtained as follows:

$$
s^{\alpha_{i}} l\left\{x_{i}(t)\right\}=l\left\{N_{i}\left(t, x_{1}, \ldots, x_{n}\right)\right\} .
$$

Now by applying the inverse Laplace transform to both sides of (13) and using the convolution theorem, the following relation can be concluded:

$$
x_{i}(t)=\int_{0}^{t} \frac{(t-\tau)^{\alpha-1}}{\Gamma(\alpha)} N_{i}\left(\tau, x_{1}(\tau), x_{2}(\tau), \ldots, x_{n}(\tau)\right) d \tau
$$

Therefore,

$$
\begin{aligned}
x_{i}^{n+1}(t) & \\
= & x_{i}^{0}(t) \\
& +\int_{0}^{t} \frac{(t-\tau)^{\alpha-1}}{\Gamma(\alpha)} \\
& \quad \times N_{i}\left(\tau, x_{1}^{n}(\tau), x_{2}^{n}(\tau), \ldots, x_{n}^{n}(\tau)\right) d \tau, \quad i=1, \ldots, n .
\end{aligned}
$$

After identifying the initial approximation of $x_{i}^{0}$, the remaining approximations $x_{1}^{n}(t), n>0$ can be obtained. So that each term can be determined by previous term and the approximation of iteration formula can be entirely evaluated. Consequently, the solution may be written as

$$
x_{i}(t)=\lim _{n \rightarrow \infty} x_{i}^{n}(t), \quad i=1, \ldots n .
$$

\section{Numerical Results}

To demonstrate the effectiveness of the method we consider some systems of linear and nonlinear fractional differential equations.

Example 1. Let us consider the following system of two linear fractional differential equations:

$$
\begin{aligned}
& D_{*}^{\beta} x(t)=x(t)+y(t), \\
& D_{*}^{\gamma} y(t)=-x(t)+y(t),
\end{aligned}
$$

subjected to the initial conditions

$$
x(0)=0, \quad y(0)=1 \text {. }
$$

Applying the RVIM to (17), the result is as follows:

$$
\begin{gathered}
l\{x(t)\}=\frac{1}{s^{\beta}} l\{x(t)+y(t)\}, \\
l\{y(t)\}=\frac{1}{s^{\gamma}} l\{-x(t)+y(t)\} .
\end{gathered}
$$

Applying the inverse Laplace transform to both sides of (19) results in

$$
\begin{aligned}
& x(t)=\frac{1}{\Gamma(\beta)} \int_{0}^{t}(t-\tau)^{\beta-1}[x(\tau)+y(\tau)] d \tau, \\
& y(t)=\frac{1}{\Gamma(\gamma)} \int_{0}^{t}(t-\tau)^{\gamma-1}[-x(\tau)+y(\tau)] d \tau .
\end{aligned}
$$

Therefore, approximate solution for (20) can be readily obtained as

$$
\begin{aligned}
& x_{n+1}(t)=x_{0}(t)+\frac{1}{\Gamma(\beta)} \int_{0}^{t}(t-\tau)^{\beta-1}\left[x_{n}(\tau)+y_{n}(\tau)\right] d \tau, \\
& y_{n+1}(t)=y_{0}(t)+\frac{1}{\Gamma(\gamma)} \int_{0}^{t}(t-\tau)^{\gamma-1}\left[-x_{n}(\tau)+y_{n}(\tau)\right] d \tau,
\end{aligned}
$$

where $x_{0}(t)=0, y_{0}(t)=1$.

According to (21), after some simplification and substitution, the following sets of equations are concluded:

$$
\begin{aligned}
x_{1}(t)= & \frac{t^{\beta}}{\Gamma(\beta+1)}, \\
y_{1}(t)= & 1+\frac{t^{\gamma}}{\Gamma(\gamma+1)}, \\
x_{2}(t)= & \frac{t^{\beta}}{\Gamma(\beta+1)}+\frac{t^{2 \beta}}{\Gamma(2 \beta+1)}+\frac{t^{\gamma+\beta}}{\Gamma(\gamma+\beta+1)}, \\
y_{2}(t)= & 1+\frac{t^{\gamma}}{\Gamma(\gamma+1)}+\frac{t^{2 \gamma}}{\Gamma(2 \gamma+1)}-\frac{t^{\gamma+\beta}}{\Gamma(\gamma+\beta+1)}, \\
x_{3}(t)= & \frac{t^{\beta}}{\Gamma(\beta+1)}+\frac{t^{2 \beta}}{\Gamma(2 \beta+1)}+\frac{t^{\gamma+\beta}}{\Gamma(\gamma+\beta+1)} \\
& +\frac{t^{3 \beta}}{\Gamma(3 \beta+1)}+\frac{t^{2 \gamma+\beta}}{\Gamma(2 \gamma+\beta+1)}, \\
& +\frac{t^{3 \gamma}}{\Gamma(3 \gamma+1)}-\frac{t^{\gamma+2 \beta}}{\Gamma(\gamma+2 \beta+1)}-\frac{2 t^{2 \gamma+\beta}}{\Gamma(2 \gamma+\beta+1)} \\
y_{3}(t)= & 1+\frac{t^{2 \gamma}}{\Gamma(\gamma+1)}+\frac{t^{\gamma+\beta}}{\Gamma(\gamma+\beta+1)}
\end{aligned}
$$

Figure 1 shows the approximate solution for system (17), obtained for the values of $\beta=\gamma=1$. This is the only case for which we know the exact solution 


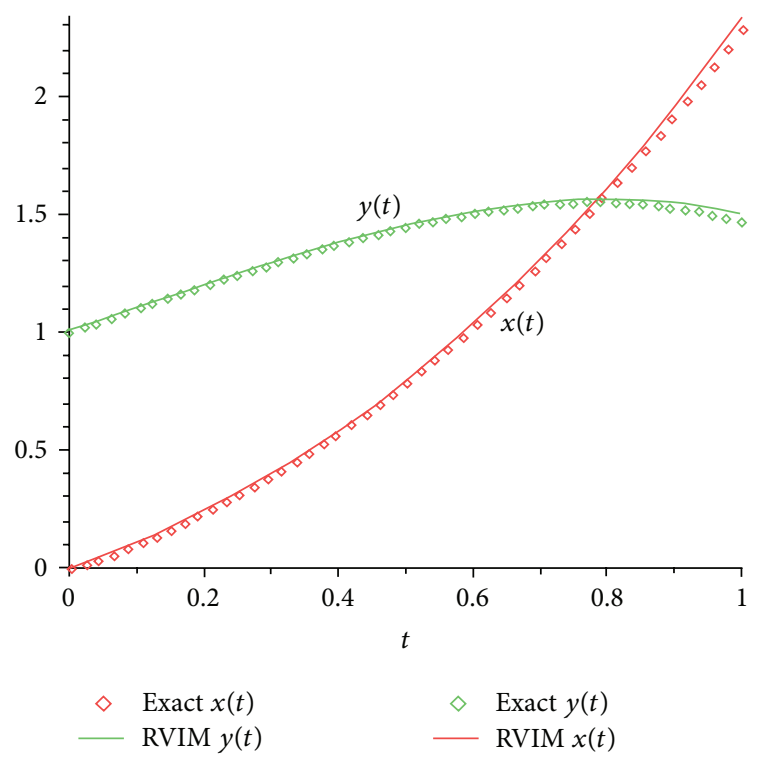

FIGURE 1: Comparison plot of RVIM method for system (17), when $\beta=\gamma=1$ with its exact solution.

$\left(x(t)=e^{t} \sin (t), y(t)=e^{t} \cos (t)\right)$. One can see that our approximate solutions by using the RVIM are in a good agreement with its exact solution.

Figure 2 shows the approximate solutions for system (17), obtained for the values of $\beta=0.7$ and $\gamma=0.9$. It is to be noted that the following three iterations were used in evaluating the approximate solution (whereas by the differential transform method twenty-five terms were used in evaluating the approximate solutions).

The results in Figures 1 and 2 are in full agreement with the results obtained in [9], using differential transform method.

Example 2. Consider the following nonlinear system of fractional differential equations:

$$
\begin{gathered}
\frac{d^{\alpha} y_{1}}{d t^{\alpha}}=-y_{1}, \\
\frac{d^{\alpha} y_{2}}{d t^{\alpha}}=y_{1}-y_{2}^{2}, \\
\frac{d^{\alpha} y_{3}}{d t^{\alpha}}=y_{2}^{2},
\end{gathered}
$$

subjected to the initial conditions

$$
y_{1}(0)=1, \quad y_{2}(0)=0, \quad y_{3}(0)=0 .
$$

Applying the RVIM to (23), the result is as follows:

$$
\begin{gathered}
l\left\{y_{1}(t)\right\}=\frac{1}{s^{\alpha}} l\left\{-y_{1}(t)\right\}, \\
l\left\{y_{2}(t)\right\}=\frac{1}{s^{\alpha}} l\left\{y_{1}(t)-y_{2}^{2}(t)\right\}, \\
l\left\{y_{3}(t)\right\}=\frac{1}{s^{\alpha}} l\left\{y_{2}^{2}(t)\right\} .
\end{gathered}
$$

Benefiting from the inverse Laplace transform to both sides of (25), one obtains

$$
\begin{gathered}
y_{1}(t)=\frac{1}{\Gamma(\alpha)} \int_{0}^{t}(t-\tau)^{\alpha-1}\left[-y_{1}(\tau)\right] d \tau, \\
y_{2}(t)=\frac{1}{\Gamma(\alpha)} \int_{0}^{t}(t-\tau)^{\alpha-1}\left[y_{1}(\tau)-y_{2}^{2}(\tau)\right] d \tau, \\
y_{3}(t)=\frac{1}{\Gamma(\alpha)} \int_{0}^{t}(t-\tau)^{\alpha-1}\left[y_{2}^{2}(\tau)\right] d \tau .
\end{gathered}
$$

Therefore, approximate solution for (26) can be readily obtained as

$$
\begin{gathered}
y_{1}^{n+1}(t)=y_{1}^{0}(t)+\frac{1}{\Gamma(\alpha)} \int_{0}^{t}(t-\tau)^{\alpha-1}\left[-y_{1}(\tau)\right] d \tau, \\
y_{2}^{n+1}(t)=y_{2}^{0}(t)+\frac{1}{\Gamma(\alpha)} \int_{0}^{t}(t-\tau)^{\alpha-1}\left[y_{1}(\tau)-y_{2}^{2}(\tau)\right] d \tau, \\
y_{3}^{n+1}(t)=y_{3}^{0}(t)+\frac{1}{\Gamma(\alpha)} \int_{0}^{t}(t-\tau)^{\alpha-1}\left[y_{2}^{2}(\tau)\right] d \tau,
\end{gathered}
$$

where $y_{1}^{0}(t)=1, y_{2}^{0}(t)=0, y_{3}^{0}(t)=0$, and $y_{i}^{n}$ indicates $n$th approximation of $y_{i}$ for $i=1,2,3$.

According to (27), after some simplification and substitution, the following set of equations is concluded:

$$
\begin{aligned}
& y_{1}^{1}(t)=1-\frac{t^{\alpha}}{\Gamma(\alpha+1)}, \\
& y_{2}^{1}(t)=\frac{t^{\alpha}}{\Gamma(\alpha+1)}, \\
& y_{3}^{1}(t)=0 \\
& y_{1}^{2}(t)=1-\frac{t^{\alpha}}{\Gamma(\alpha+1)}+\frac{t^{2 \alpha}}{\Gamma(2 \alpha+1)}, \\
& y_{2}^{2}(t)=\frac{t^{\alpha}}{\Gamma(\alpha+1)}-\frac{t^{2 \alpha}}{\Gamma(2 \alpha+1)}-\frac{\Gamma(2 \alpha+1) t^{3 \alpha}}{(\Gamma(\alpha+1))^{2} \cdot \Gamma(3 \alpha+1)}, \\
& y_{3}^{2}(t)=\frac{\Gamma(2 \alpha+1) t^{3 \alpha}}{(\Gamma(\alpha+1))^{2} \cdot \Gamma(3 \alpha+1)}, \\
& y_{1}^{3}(t)=1-\frac{t^{\alpha}}{\Gamma(\alpha+1)}+\frac{t^{2 \alpha}}{\Gamma(2 \alpha+1)}, \\
& y_{2}^{3}(t)=\frac{t^{\alpha}}{\Gamma(\alpha+1)}-\frac{t^{2 \alpha}}{\Gamma(2 \alpha+1)}-\frac{\Gamma(2 \alpha+1) t^{3 \alpha}}{(\Gamma(\alpha+1))^{2} \cdot \Gamma(3 \alpha+1)}, \\
& y_{3}^{3}(t)=\frac{\Gamma(2 \alpha+1) t^{3 \alpha}}{(\Gamma(\alpha+1))^{2} \cdot \Gamma(3 \alpha+1)} .
\end{aligned}
$$




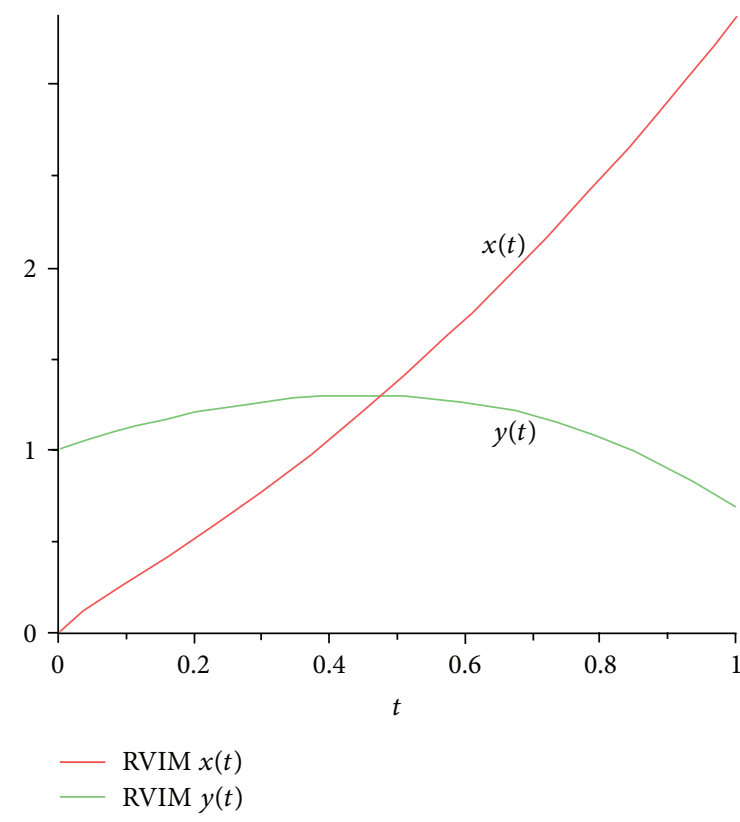

(a)

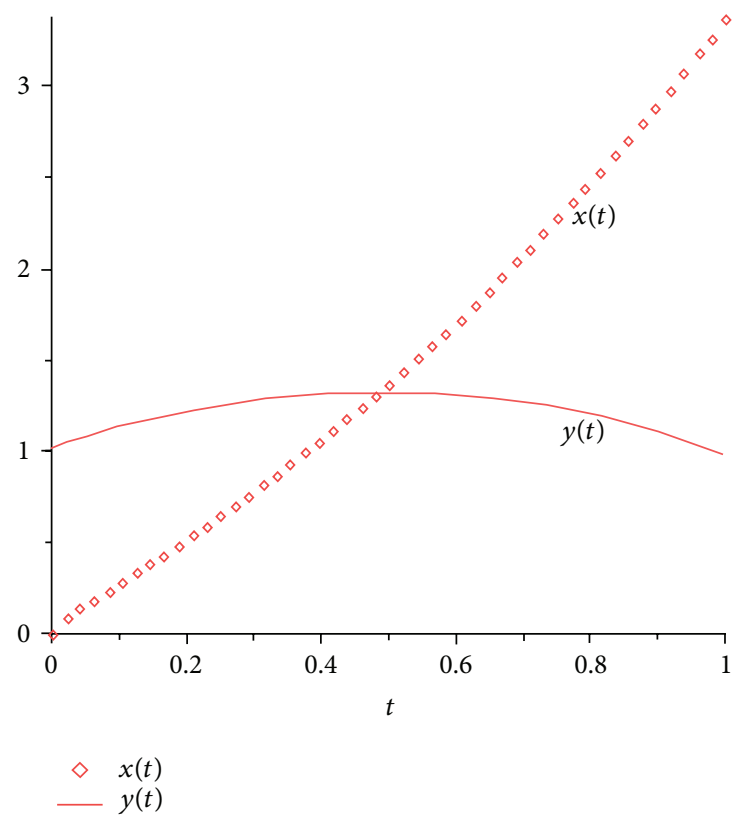

(b)

FIGURE 2: Plots of system (17), when $\beta=0.7, \gamma=0.9$ : (a) RVIM method and (b) differential transform method.

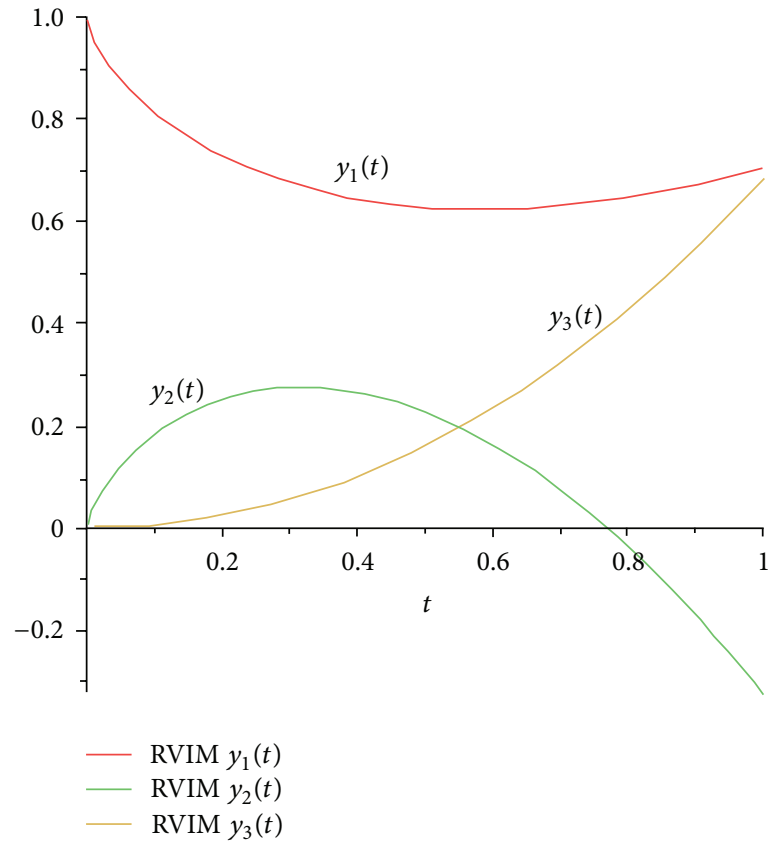

(a)

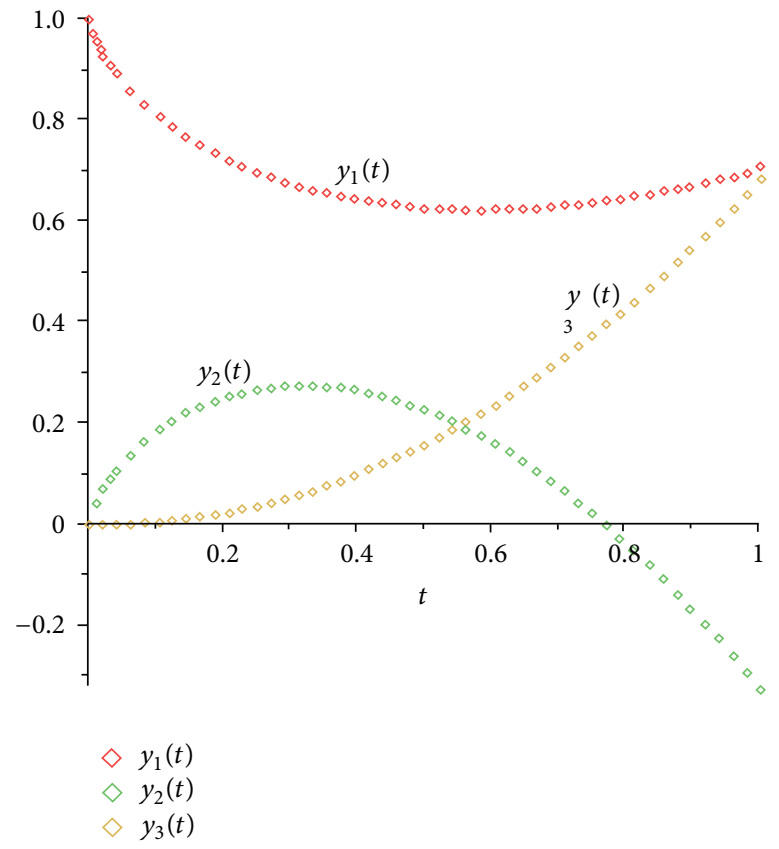

(b)

FIGURE 3: Plots of system (23): (a) RVIM method and (b) differential transform method.

It is to be noted that we reached the approximate solution after three iterations by the method of RVIM, whereas it is obtained after seventy iterations by the differential transform method. In Figure 3, we draw the curves of approximate solutions $y_{1}(t), y_{2}(t)$, and $y_{3}(t)$, which is obtained for the value of $\alpha=0.7$. The graphical results are in a very good agreement with the results in [9].
Example 3. Lastly we consider the following system of two nonlinear fractional differential equations:

$$
\begin{aligned}
& D_{*}^{1.3} x(t)=x(t)+y^{2}(t), \\
& D_{*}^{2.4} y(t)=x(t)+5 y(t),
\end{aligned}
$$




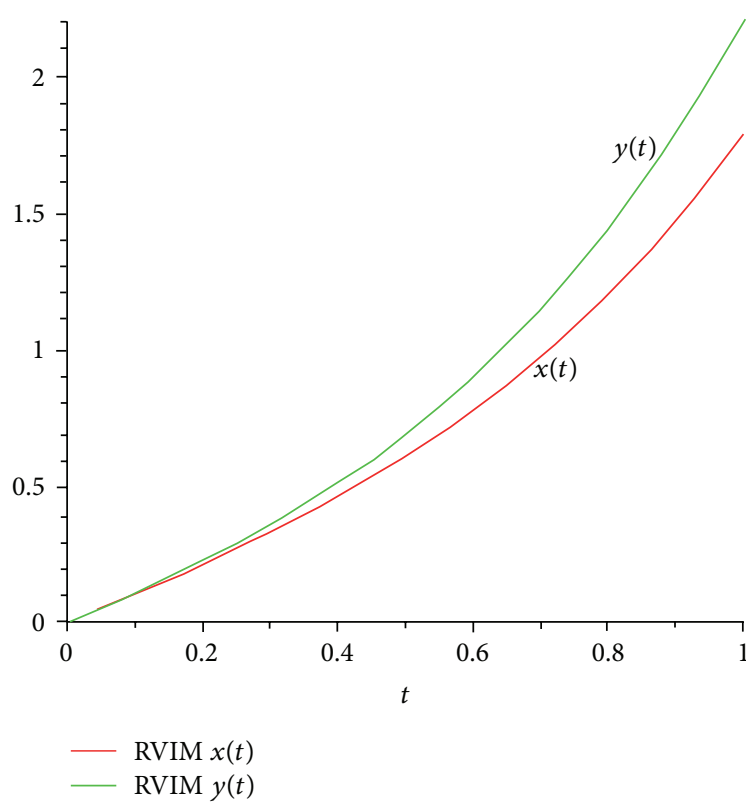

(a)

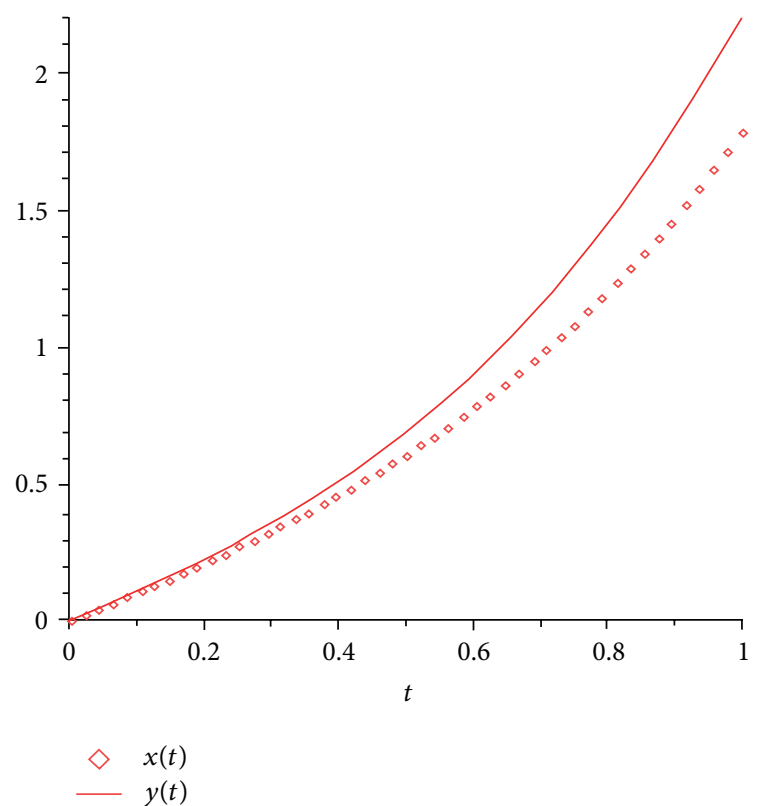

(b)

FIgURE 4: Plots of system (29): (a) RVIM method and (b) differential transform method.

with the initial conditions

$$
\begin{gathered}
x(0)=0, \quad x^{\prime}(0)=1, \\
y(0)=0, \quad y^{\prime}(0)=1, \quad y^{\prime \prime}(0)=1 .
\end{gathered}
$$

Applying the RVIM to (29), the result is as follows:

$$
\begin{aligned}
& l\{x(t)\}=\frac{1}{s^{1.3}} l\left\{x(t)+y^{2}(t)\right\}, \\
& l\{y(t)\}=\frac{1}{s^{2.4}} l\{x(t)+5 y(t)\} .
\end{aligned}
$$

Benefiting from the inverse Laplace transform to both sides of (31), one obtains

$$
\begin{gathered}
x(t)=\frac{1}{\Gamma(1.3)} \int_{0}^{t}(t-\tau)^{0.3}\left[x(\tau)+y^{2}(\tau)\right] d \tau, \\
y(t)=\frac{1}{\Gamma(2.4)} \int_{0}^{t}(t-\tau)^{1.4}[x(\tau)+5 y(\tau)] d \tau .
\end{gathered}
$$

Therefore, approximate solution for (32) can be readily obtained as:

$$
\begin{aligned}
& x_{n+1}(t)=x_{0}(t)+\frac{1}{\Gamma(1.3)} \int_{0}^{t}(t-\tau)^{0.3}\left[x(\tau)+y^{2}(\tau)\right] d \tau, \\
& y_{n+1}(t)=y_{0}(t)+\frac{1}{\Gamma(2.4)} \int_{0}^{t}(t-\tau)^{1.4}[x(\tau)+5 y(\tau)] d \tau,
\end{aligned}
$$

where the initial approximation must be satisfied by the following equations:

$$
x_{0}(t)=t, \quad y_{0}(t)=t+\frac{t^{2}}{2} .
$$

According to (33), after some simplification and substitution, the following sets of equations are concluded:

$$
\begin{gathered}
x_{1}(t)=t+\frac{t^{2.3}}{\Gamma(3.3)}+\frac{2 t^{3.3}}{\Gamma(4.3)}+\frac{6 t^{5.3}}{\Gamma(6.3)}+\frac{6 t^{4.3}}{\Gamma(5.3)}, \\
y_{1}(t)=t+\frac{t^{2}}{2}+\frac{6 t^{3.4}}{\Gamma(4.4)}+\frac{5 t^{4.4}}{\Gamma(5.4)} .
\end{gathered}
$$

Figure 4 shows the efficiency of this method to obtain approximate solutions of system (29).

\section{Conclusion}

In this paper, Reconstruction of Variational Iteration Method (RVIM) was successfully employed to solve systems of differential equations of fractional order. The work emphasized our belief that the method is a reliable technique to handle linear and nonlinear systems of fractional differential equations.

The results of this method are in a good agreement with those obtained by using the differential transform method and the Adomian decomposition method. One of the advantages of this method in comparison with the Adomian decomposition method is that we do not need to do the difficult computation for finding the Adomian polynomials.

Moreover, the method presented rapidly convergent successive approximations without any restrictive assumptions or transformation which may change the physical behavior of the problem.

Evidently, the RVIM reduced the size of calculation and also the iteration was direct and straight forward.

Generally, the proposed method is promising and applicable to a board class of linear and nonlinear systems in the theory of fractional calculus. 


\section{References}

[1] F. Mainardi, "Fractional calculus: some basic problems in continuum and statistical mechanics," in Fractals and Fractional Calculus in Continuum Mechanics, vol. 378, pp. 291-348, Springer, New York, NY, USA, 1997.

[2] A. Carpinteri and F. Mainardi, Fractals and Fractional Calculus in Continuum Mechanics, Springer-Verlag, New York, NY, USA, 1997.

[3] R. Gorenflo and F. Mainardi, "Fractional calculus: integral and differential equations of fractional order," in Fractals and Fractional Calculus in Continuum Mechanics, vol. 378, pp. 223276, Springer, New York, NY, USA, 1997.

[4] A. Dold and B. Eckmann, Fractional Calculus and Its Applications, Springer, Berlin, Germany, 1975.

[5] G. Adomian, "A review of the decomposition method and some recent results for nonlinear equations," Mathematical and Computer Modelling, vol. 13, no. 7, pp. 17-43, 1990.

[6] J.-S. Duan and R. Rach, "A new modification of the Adomian decomposition method for solving boundary value problems for higher order nonlinear differential equations," Applied Mathematics and Computation, vol. 218, no. 8, pp. 4090-4118, 2011.

[7] M. Kumar and N. singh, "Modified the Adomian decomposition method and computer implementation for solving singular boundary value problems arising in various physical problems," Computer and Chemical Engineering, vol. 34, pp. 1750-1760, 2010.

[8] H. Jafari and V. Daftardar-Gejji, "Solving a system of nonlinear fractional differential equations using Adomian decomposition," Journal of Computational and Applied Mathematics, vol. 196, no. 2, pp. 644-651, 2006.

[9] V. S. Ertürk and S. Momani, "Solving systems of fractional differential equations using differential transform method," Journal of Computational and Applied Mathematics, vol. 215, no. 1, pp. 142-151, 2008.

[10] J. H. He, "Variational iteration method-a kind of nonlinear analytical technique: some examples," International Journal of Non-Linear Mechanics, vol. 34, pp. 699-708, 1999.

[11] J. K. Zhou, Differential Transformation and its Applications for Electrical Circuits, Huazhong University Press, Wuhan, China, 1986, (Chinese).

[12] E. Hesameddini and H. Latifizadeh, "Reconstruction of variational iteration algorithm using the Laplace transform," Iternational Journal of Nonlinear Sciences and Numerical Simulation, vol. 10, pp. 1365-1370, 2009. 


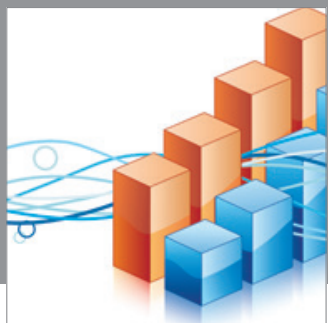

Advances in

Operations Research

mansans

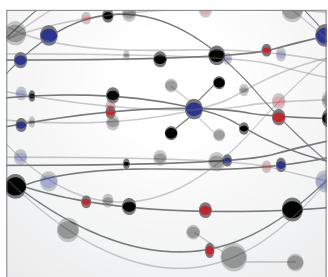

The Scientific World Journal
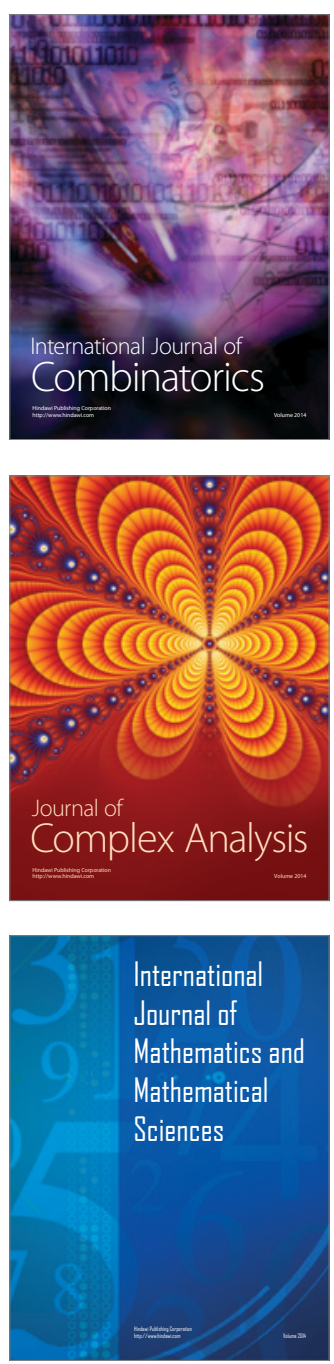
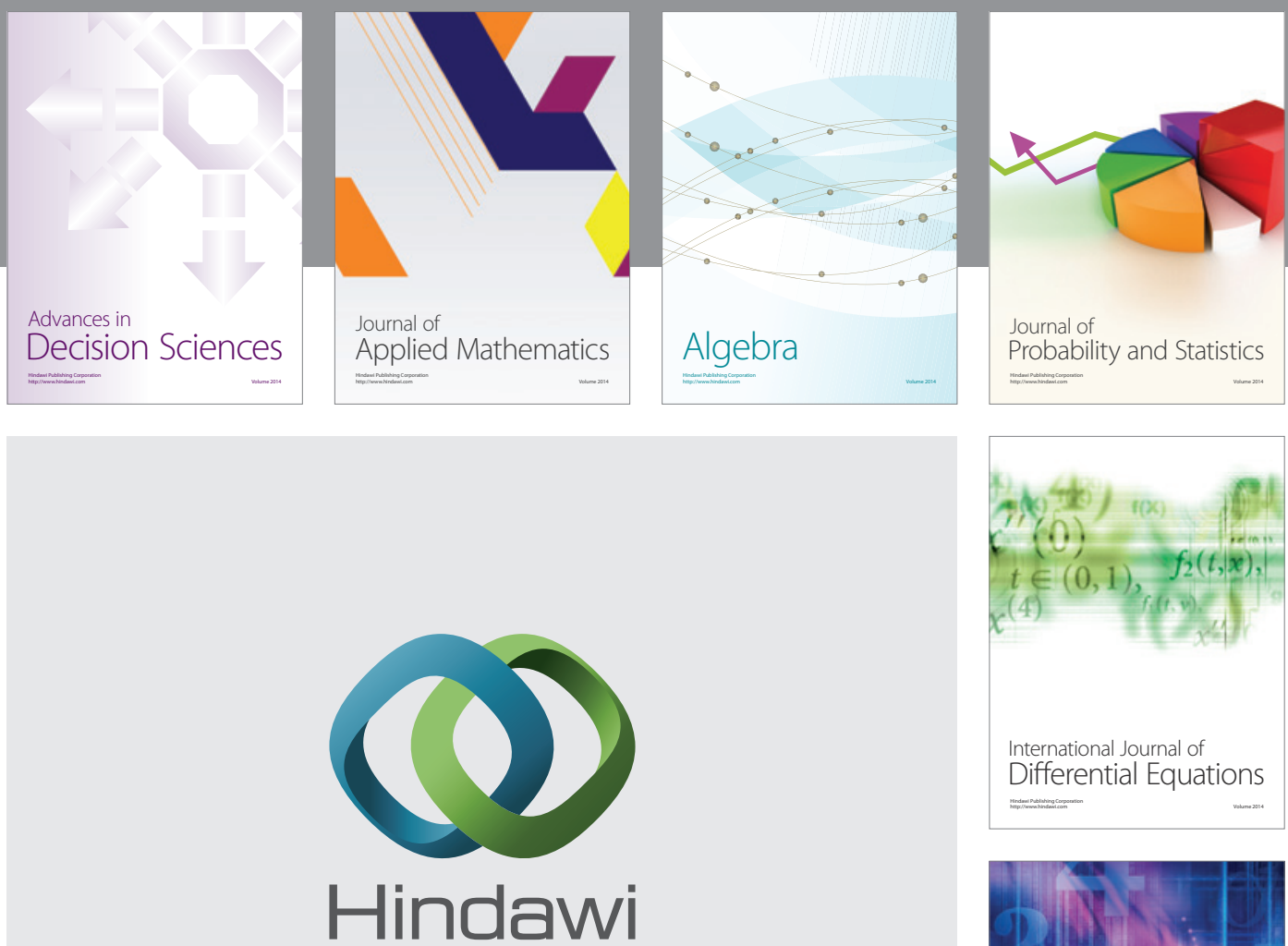

Submit your manuscripts at http://www.hindawi.com
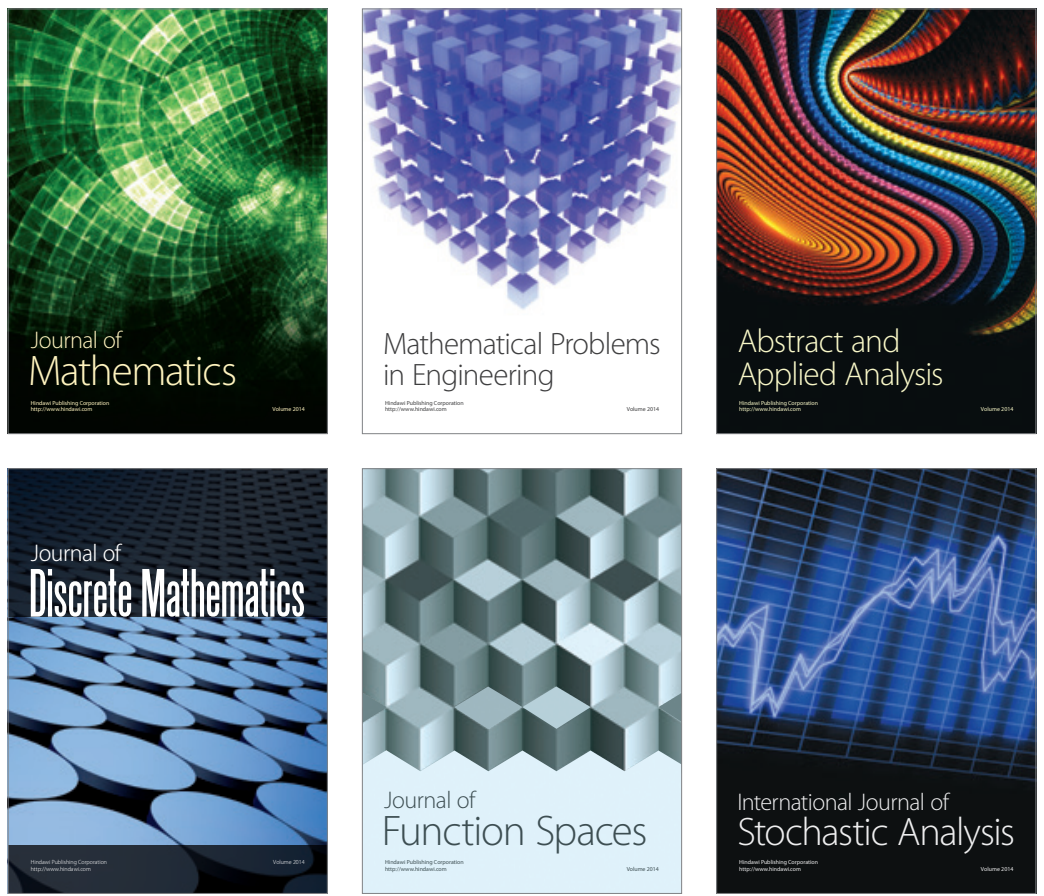

Journal of

Function Spaces

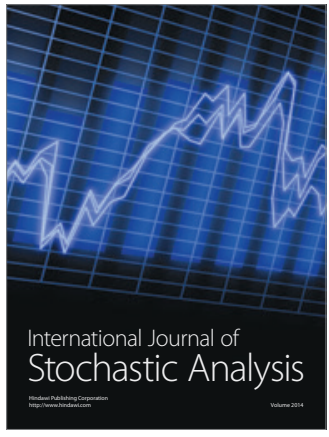

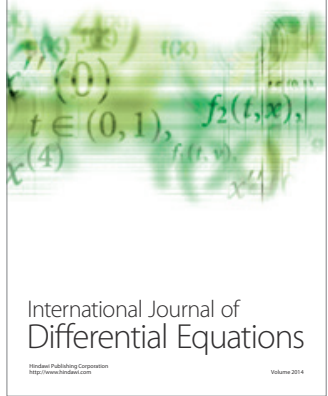
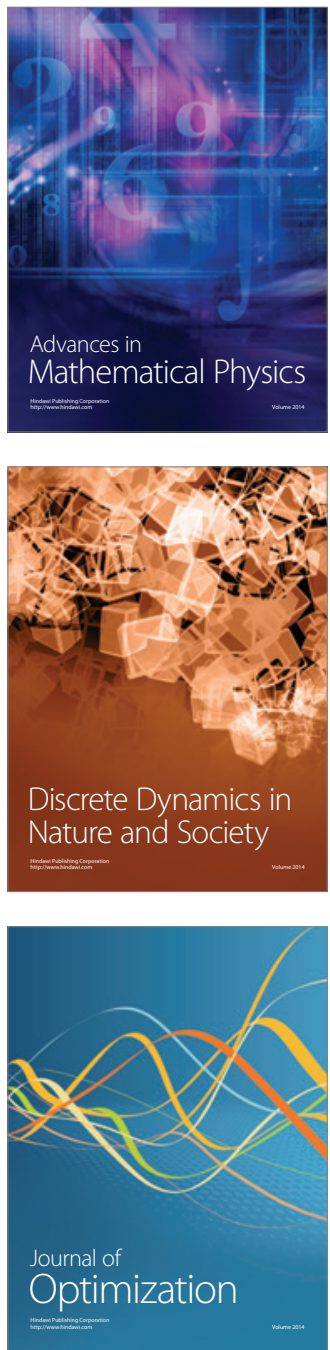